\title{
Control Unit for Power Subsystem of a Wireless Sensor Node
}

\author{
Michal Kochláň, IEEE Student Member \\ Department of Technical Cybernetics \\ Faculty of Management Science and Informatics \\ University of Žilina \\ Univerzitná 8215/1, 01026 Žilina, Slovakia \\ Email: michal.kochlan@fri.uniza.sk
}

\author{
Samuel Žák, Juraj Miček, Jana Milanová \\ Department of Technical Cybernetics \\ Faculty of Management Science and Informatics \\ University of Žilina \\ Univerzitná 8215/1, 01026 Žilina, Slovakia \\ Email: \{samuel.zak, juraj.micek, jana.milanova\}@fri.uniza.sk
}

\begin{abstract}
This paper addresses analysis of the principle of a bi-directional electric energy charge pump and describes circuit solution for control unit of a wireless sensor node power subsystem. This power and control unit, as the power subsystem of the wireless node, comprises two supercapacitors. Bi-directional energy flow between these two supercapacitors takes place in order to optimally satisfy the sensor node energy requirements. At the same time, the bi-directional energy flow enables optimal energy harvesting from the selected energy scavenging (harvesting) subsystem. The mentioned Power Unit Control System has been successfully verified in the process of energy harvesting from a solar module.
\end{abstract}

\section{INTRODUCTION}

$\mathbf{I}$ N RECENT years, we encounter with many new unconventional applications of wireless sensor networks (WSNs). Related to the expected rapid expansion of so called Internet of Everything (IoE) applications [1], [2], [3], the problem of WSN is about to stand in the center of scientists community interest as well as of the public.

Basic limiting factors of WSN expansion are price and unattended operation time [4]. Both mentioned limitations are closely related to the energy requirements of essential nodes of a sensor network. The issue of effective sensor node power supply is being solved by two basic methods [5], [6]:

- Reducing a sensor node (SN) energy requirements,

- Consistent utilization of energy harvesting (EH) options from sources that are available in given application.

Both methods are used these days. Modern low power components are used for construction of present SNs, which help to reduce the energy requirements. In running mode, the SN uses modes with reduced power consumption and their combinations [7]. This approach turns MCU into active mode only in case the application or (external) conditions of monitored environment require it. Second option is the utilization of EH systems that are designed so that they would be able to ensure SN operation [8]. Today, there is a popular term Zero Power Wireless Sensor [9], which represents just solutions that do not need power supply for continuous operation, but they are able to harvest required energy from the environment.

This work was supported by Centre of excellence for systems and services of intelligent transport I. and Research Centre of University of Žilina.
However, until recently, the lifetime of such solutions was limited to finite battery life or the number of charging cycles [10]. These limitations have been overcome by supercapacitors since they are characterized by high number of charging cycles (up to 1 million) [21]. Combination of low-power integrated circuits, effective systems of energy transformation from environment to electric energy and advanced tools for electric energy accumulation enables pushing the boundaries of WSN applications to the areas (mostly applications of information and communication means), where no one would have imagined their effective operation few years ago.

\section{SEnsor Node}

Sensor networks consist of large number of sensor nodes (SNs). The SNs are deployed in a sensor field, where each of these sensor nodes has the capabilities to collect and route data to the sink and the end users [12]. In order the basic characteristics of a sensor network could be satisfied, each SN has to perform the following functions [13]:

- Data collection,

- Data preprocessing,

- Communication.

It is obvious that except the mentioned basic functions of each network node, there are also other important SN parameters, such as [14]:

- Computing performance of the sensor node,

- Low power consumption/long lifetime,

- Production cost,

- Security,

- Fault tolerance.

Many of mentioned requirements are contradicting each other [12]. Increasing computing performance increases energy requirements as well as sensor price. Enhancing transmission security involves higher computing performance. Higher fault tolerance usually negatively influences production cost. Therefore, it is impossible to develop appropriately universal node, which would be optimal in terms of all mentioned requirements. Moreover, it should be noted that different applications of SNs set different weight for the mentioned requirements. Problem of sensing elements designing is described in 
[15]. The authors propose to use class of universally usable modules for development and application-oriented carriers and, thus, reach high degree of universality with possibilities to use the developed systems in the widest set of various applications. Data collection is performed by the data collection subsystem of a sensor node. This subsystem is extremely dependent on the requirements of the particular application and consist of sensing elements observing parameters that are relevant for solving the desired task. It is clear that different sensing elements are required for road traffic monitoring, other for patient telemonitoring and absolutely another for environment monitoring.

Because the energy requirements for data transmission are very high [5], it is suitable to perform significant part of data processing process directly at place of acquisition - in the sensor node. Then, the other network nodes will transfer only data that contain substantial information for solving the given problem.

It is clear, that in each sensor node data compression is executed. Then, the fundamental processes of data pre-processing relate to extraction methods of information content. Not in all WSN applications is the sensor node's task relegated only to the operations related to signal compression, increasingly there are used Collaborative Signal Processing Algorithms [16], that use suitable distribution of partial task among each nodes in order to increase the computing performance of network while minimizing of whole energy consumption. Today there is a wide range of various methods that can be successfully implemented onto WSN platform (Kalman filtering, neural networks, Bayesian inference etc.) [17].

Wireless communication ability is one of the basic features of a sensor node [5]. Communication abilities of WSN nodes are in most cases limited by communication range and transmission speed. It should be reminded that with increasing communication range the transmission power/sensitivity raises. This always leads to increased requirements on energy consumption.

Based on the mentioned functions and parameters of the $\mathrm{SN}$, it is possible to create a generalized schematic block of a sensor node, which is depicted on Fig. 1. Each WSN node contains four basic units [4]:

- Sensing unit,

- Processor unit,

- Communication unit,

- Power unit.

In terms of WSN utilization properties, operating time of the network without need of operator intervention is important. The critical point is just the power supply of the SN. As mentioned earlier, the unit that takes care of power supply for a sensor node is called power unit. This part of sensor node can be simply represented by a power system with use of primary (battery) cells, or it may be constructed of a complex power supply system with energy harvesting $(\mathrm{EH})$ features with effective energy accumulation. The following section describes the power unit developed at our department.

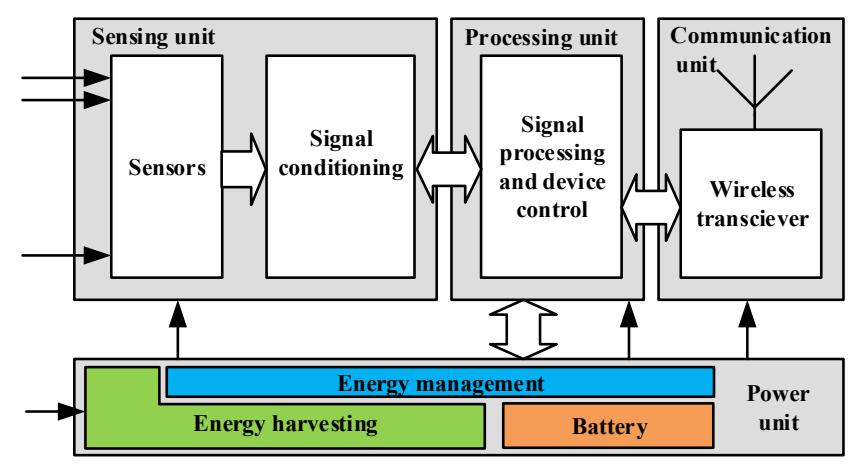

Fig. 1. Wireless node

\section{EnERgy MAnAgement System}

Ensuring the continuous effective operation of a sensor node, which has limited energy sources is a difficult problem. Solving this consists of:

- Right choice of EH type,

- Right choice of energy storage capacity,

- Designing such sensor node operation mode that minimizes energy consumption.

Subsystem that covers energy requirements of a sensor node is power unit. The power unit can be implemented in several ways. Today we encounter very simple power supply systems using primary (battery) cells that are designed so that the energy requirements and desired SN lifetime are met.

\section{A. Power Unit}

Power supply of a SN is realized through the subsystem, which is often referred as power unit. This supply subsystem can be based on one of the following options [18]:

- Supply from primary (battery) cells,

- Supply from rechargeable batteries,

- Energy harvesting (EH) features,

- Near Field Charging (NFC).

Today one can often meet complex solutions for power units that satisfy energy management in such way that all WSN energy requirements are compressed down to zero (zero power systems) [19]. Such unit consists of three main parts, Fig. 2:

- Energy harvesting system,

- Energy processor,

- Energy storage.

Functions of each subsystem of power unit result from their names. $\mathrm{EH}$ is used to temporary preserve energy and energy management controls the operation of the whole power unit in the way that it is possible to ensure all requirements of a sensor node with reaching the maximal energy effectiveness.

Unless the application permits performing basic maintenance during sensor node lifetime - battery exchange, then rechargeable batteries are often used. In those simple cases is energy consumption control (transitions to energy saving modes of individual subsystems) often performed by the SN processing unit. In case more complex power units are used 
TABLE I

SELECTED PARAMETERS OF ENERGY STORAGE SYSTEMS $(1 \mathrm{KWH}=3,6 \mathrm{MJ})$

\begin{tabular}{|c|c|c|c|c|c|}
\hline \multicolumn{2}{|c|}{ Type } & Specific energy $[\mathrm{MJ} / \mathrm{kg}]$ & Number of cycles $\mathbf{N}$ & Self discharge rate [\%] & Charge/discharge Efficiency [\%] \\
\hline \multirow{2}{*}{ Primary batteries } & Alkaline & 0.6 & - & 3 per year & - \\
\hline & Lithium & 2 & - & 2 per year & - \\
\hline \multirow{4}{*}{$\begin{array}{l}\text { Rechargeable } \\
\text { (liquid } \\
\text { electrolyte) }\end{array}$} & Lead Acid & 0.14 & $500-800$ & 3-20 per month & $50-90$ \\
\hline & NiMH & 0.36 & $500-1800$ & 8 per month & 66 \\
\hline & Li-Ion & 0.9 & $400-1200$ & 5 per month & $80-90$ \\
\hline & Li-Poly & 0.95 & $500-1000$ & 8 per month & $80-90$ \\
\hline $\begin{array}{l}\text { Rechargeable } \\
\text { (solid electrolyte) }\end{array}$ & $\begin{array}{l}\text { ss-battery } \\
\text { EnerChip }\end{array}$ & 1.8 & 5000 & 2 per year & 90 \\
\hline Supercapacitors & Twolayers & $0.01-0.02$ & 500000 & 50 per week & 95 \\
\hline $\begin{array}{l}\text { Pseudosuper } \\
\text { capacitors }\end{array}$ & Li-Ion & 0.055 & 10000 & 10 per month & 90 \\
\hline
\end{tabular}

Sensor node

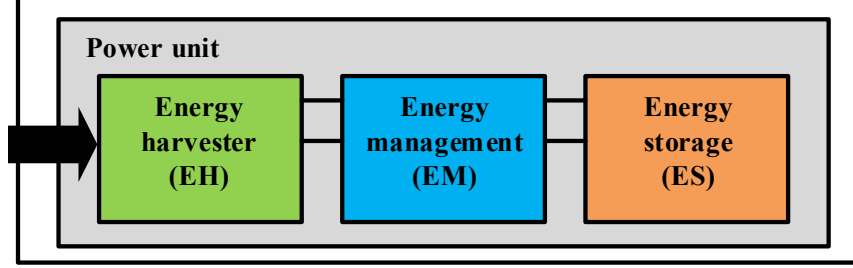

Fig. 2. Power unit

(combined energy storage and energy harvesting, etc.) the control of individual subsystems is often performed by a specialized unit - energy processor. When designing the energy management (EM) system, it is possible to use some of commercially available solutions. As an example, it is possible to mention energy processor CBC915 [20]. Other possibility is to create the Power management unit based on Low power MCU. That solution is universal enough and usable for wide range of applications with low-power energy consumption.

\section{B. Energy Storages}

Energy storage (ES) is one of the basic parts of power unit. It is used for energy storage that is used for SN supply. ES accumulates the electric energy and delivers it to the powered device. In case the EH systems is used and that is not able to generate sufficient amount of energy from its environment, the ES temporarily helps EH subsystem with delivering enough power. It is clear, that either case (using EH or not) there is always need for energy to be accumulated and preserved in some way. In present, there are usually used the following "media" for energy accumulation:

- Primary batteries,

- Rechargeable batteries,

- Solid state batteries,

- Supercapacitors.

Selected parameters of above listed options of energy storages are described in Table I for comparison. Note, that in terms of long-term node operation, particularly the number of charge/discharge cycles is important, partially also a selfdischarge rate, but in terms of continuous operation of node with EH system utilization energy efficiency is important. Therefore, it is clear that there is no commonly valid criterion, which recommends specific type of energy storage. Selection is always dependent on specific conditions and nature of the application.

One of the parameters of modern energy storage systems is specific energy (energy density), which moves from $2 \mathrm{MJ} / \mathrm{kg}$ with high-end primary lithium batteries up to $0.01 \mathrm{MJ} / \mathrm{kg}$ with supercapacitors [18]. Specific energy of primary lithium batteries is up to 100-times higher then current supercapacitors can reach. But specific energy is not the only parameter for choosing suitable type of energy storage. Not the less important parameters are number of charging cycles, self discharge rate, operating conditions, etc.

Because the parameters and limitations of each battery type vary quite much, there are often combinations of various types of elements for energy storage. In many applications it is possible to preferably use the combination of supercapacitor and rechargeable battery. For example, this combination benefits from high number of charge/discharge cycles of a supercapacitor and high specific energy of a battery. Especially, this combination appears a lot in power units based on $\mathrm{EH}$.

\section{Energy Harvesting}

$\mathrm{EH}$ represents ability to obtain the energy from natural resources in environment and transforms it to the electric energy [6]. Ambient energy, that can be used for power supply of low-power electronic systems is in various forms. It depends on the specific application, which takes the available energy in a suitable form. Common types of ambient energy sources, which is possible to use in EH systems for WSN are [21]:

- Biochemical energy,

- Mechanical energy,

- Acoustic noise energy,

- Wind energy,

- Thermal energy,

- Photovoltaic energy,

- Wireless energy. 
It is obvious that it is quite impossible to define generally applicable rule, which type of energy is optimal for utilization in WSN. Choice of power source type depends on specific requirements of the $\mathrm{SN}$ and environment possibilities, which the node operates in. Basic comparison of possibilities that can be used when designing $\mathrm{EH}$ system are summarized in Table ??

TABLE II

POWER DENSITY OF EH TECHNIQUe

\begin{tabular}{|l|l|}
\hline \multicolumn{1}{|c|}{ Energy harvesting technique } & Power density \\
\hline Photovoltaic & $\begin{array}{l}\text { Outdoors (sunny day) } \\
15 \mathrm{~mW} / \mathrm{cm}^{2} \\
\text { Indoors } 10 \mu \mathrm{W} / \mathrm{cm}^{2}\end{array}$ \\
\hline Thermal energy - Thermoelectric & $\begin{array}{l}\text { Human } 30 \mu \mathrm{W} / \mathrm{cm}^{2} \\
\text { Industrial } 110 \mathrm{~mW} / \mathrm{cm}^{2}\end{array}$ \\
\hline Mechanical energy - Piezoelectric & $250 \mu \mathrm{W} / \mathrm{cm}^{3}$ \\
\hline Mechanical energy - Electromagnetic & Human $1-4 \mu \mathrm{W} / \mathrm{cm}^{3}$ \\
& Industrial $800 \mu \mathrm{W} / \mathrm{cm}^{3}$ \\
\hline Mechanical energy - Electrostatic & $50100 \mu \mathrm{W} / \mathrm{cm}^{3}$ \\
\hline Wireless energy - RF radiation & $\mathrm{GSM} 0.1 \mu \mathrm{W} / \mathrm{cm}^{2}$ \\
& $\mathrm{WiFi} 0.01 \mu \mathrm{W} / \mathrm{cm}^{2}$ \\
\hline Wind energy & $380 \mu \mathrm{W} / \mathrm{cm}^{3} @ 5 \mathrm{~m} / \mathrm{s}$ \\
\hline Acoustic noise & $0.96 \mu \mathrm{W} / \mathrm{cm}^{3} @ 100 \mathrm{~dB}$ \\
& $0.003 \mu \mathrm{W} / \mathrm{cm}^{3} @ 75 \mathrm{~dB}$ \\
\hline
\end{tabular}

In WSN applications with EH systems photovoltaic energy, thermal energy and mechanical energy are most frequently used. In present, there are appearing always new systems of effective energy conversion from environment to electric energy, which encourages the development of new, unconventional solutions.

\section{Principled Design of Proposed Power Unit MANAGEMENT SYSTEM}

In the context of increasing of capacity elements for storing electric energy (supercapacitors) new applications arise, in which traditional solutions that use rechargeable or primary sources are replaced by supercapacitors. A solution for efficient energy flow between supercapacitors $C 1$ and $C 2$ or rechargeable cells is shown in schematic Figure 5. First, let's have a look at the principle of the energy flow and then the technical solution will be presented.

\section{A. Energy flow from $\mathrm{Cl}$ to $\mathrm{C} 2$ (direct mode)}

The description of the basic function comes out from the assumption that the voltage on the capacitor $C 1$ is within the range of permissible working voltage of the used control circuit (CC).

$\mathrm{CC}$ is capable of $C 1$ capacitor voltage monitoring via $A D C 1$ and $A D C 2$, and similarly on the secondary capacitor $C 2$. In case it is necessary to transfer energy from the capacitor $C 1$ to the capacitor $C 2, \mathrm{CC}$ closes the switch $S 1$ through $D O 1$ for time $T 11$. Immediately after the time $T 11$ switch $S 1$ closes and opens switch $S 2$ for time $T 21$. The energy stored in the inductance $L$ at the time $T 21$ is transferred to the capacitor $C 2$. Waveforms of control signals of switches $S 1$ and $S 2$ are shown in Figure 3.

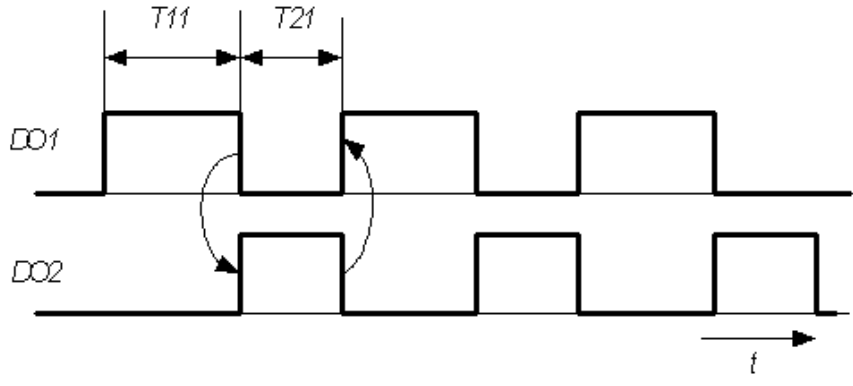

Fig. 3. Control signals in direct mode

Time $T 11$ can be calculated with the following assumptions:

- Assume that the inductance coil $L$ is used. Let the $I_{L_{\max }}$ be the maximum current that is the inductance coil able to handle;

- The voltage $U 1$ denotes voltage at capacitor $C 1$ and the voltage on the capacitor $C 2$ is labeled $U 2$.

Then for time $T 11$ applies:

$$
T 11 \leq \frac{L \cdot I_{L_{\max }}}{U_{1}}
$$

For time $T 21$ applies the following:

$$
T 21 \leq \frac{U_{1}}{U_{2}} \cdot T 11
$$

Please note that from (1) arises the fact that for times higher than $T 11$ there is a current overload of inductance $L$. At higher times than the switch-on time $T 21$ there is a discharge of supercapacitor $C 2$, which results from (2), and thereby reduces the efficiency of the device.

Further, the $\mathrm{CC}$ has up-to-date information about the $U 1$ and $U 2$ voltages through an integrated ADC peripheral. On the basis of equations (1) and (2) switching times T11 and $T 21$ can be optimally calculated by CC.

Essential amount of energy transferred in one cycle can be expressed by the following formula:

$$
\Delta E=\frac{1}{2} C 1 \cdot U_{1}^{2}\left(1-\cos \left(\frac{T 11}{\sqrt{L \cdot C 1}}\right)\right) .
$$

Eventually, in simplified form where $T 11<<\sqrt{L C 1}$ applies, equation (3) turns into:

$$
\Delta E=\frac{1}{4} \frac{U_{1}^{2} \cdot T 11^{2}}{L} .
$$

Remark 4.1: Simultaneous opening of switches $S 1$ and $S 2$ leads to the destruction of the circuit, therefore, it should be strictly taken to comply with equations 1 and 2 . These equations are approximate relations, while assuming that the opening time $T 11<<\sqrt{L C 1}$ and $T 21<<\sqrt{L C 2}$ with sufficient accuracy to satisfy the requirements of practical implementation. 


\section{B. Energy flow from C2 to C1 (inverse mode)}

In case it is necessary to transfer the energy in the opposite direction than described in the previous subsection - from capacitor $C 2$ to the capacitor $C 1$ - the switching mode of switches $S 1$ and $S 2$ is similar to the previous mode, except that the order of switching is reversed. In this case, CC through $D O 2$ closes at first switch $S 2$ for time $T 22$ and after that the switch $S 2$ is closed and switch $S 1$ opens for time $T 12$. The energy stored in the inductance $L$ is during time $T 12$ transfered to capacitor $C 1$. Waveforms of control signals of switches $S 1$ and $S 2$ are shown in Figure 4.

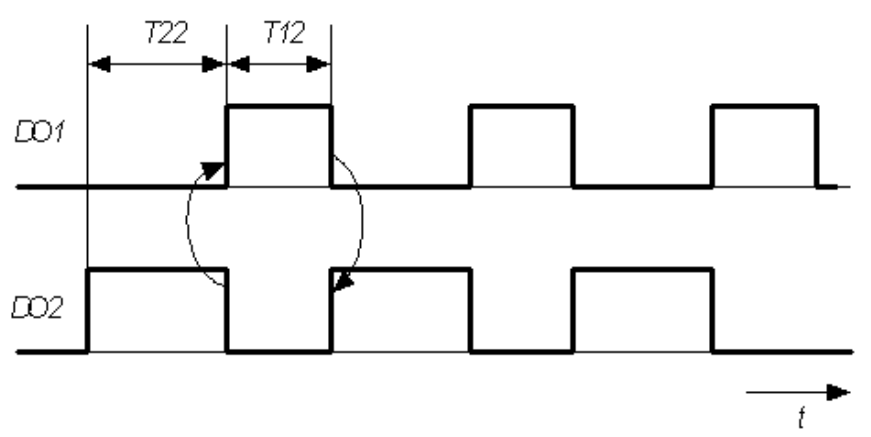

Fig. 4. Control signals in inverse mode

Similarly to the previous case, the conditions for calculating time $T 22$ are as follows:

- Assume that the inductance coil $L$ is used. Let the $I_{L_{\max }}$ be the maximum current that is the inductance coil able to handle;

- The voltage $U 1$ denotes voltage at capacitor $C 1$ and the voltage on the capacitor $C 2$ is labeled $U 2$.

Then for time $T 22$ applies:

$$
T 22 \leq \frac{L \cdot I_{L_{\max }}}{U_{2}} .
$$

For time $T 12$ applies the following:

$$
T 12 \leq \frac{U_{2}}{U_{1}} \cdot T 22 .
$$

Even in inverse mode, the similar restrictions. Therefore, please note that from (5) arises the fact that for times higher than $T 22$ there is a current overload of inductance $L$. At higher times than the switch-on time $T 12$ there is a discharge of supercapacitor $C 1$, which results from (6), and thus reduces the efficiency of the device.

Further, the $\mathrm{CC}$ has up-to-date information about the $U 1$ and $U 2$ voltages through an integrated ADC peripheral. On the basis of equations (5) and (6) switching times T22 and $T 12$ can be optimally calculated by CC.

Remark 4.2: Simultaneous opening of switches $S 1$ and $S 2$ leads to the destruction of the circuit, therefore, it should be strictly taken to comply with figures 5 and 6 . These equations are approximate relations, while assuming that the opening time $T 22<<\sqrt{L C 2}$ and $T 12<<\sqrt{L C 1}$ with sufficient accuracy to satisfy the requirements of practical implementation.

\section{Technical Solution of Proposed Power Unit MANAGEMENT SYSTEM}

The previous section describes the proposed power unit principle of operation - energy flow. This device controls power transmission with EH system, supercapacitor and rechargeable battery or supercapacitor, its principle schematic is depicted in Fig. 5. Please note that some parts that are not substantial in terms of function are not mentioned in the figure.

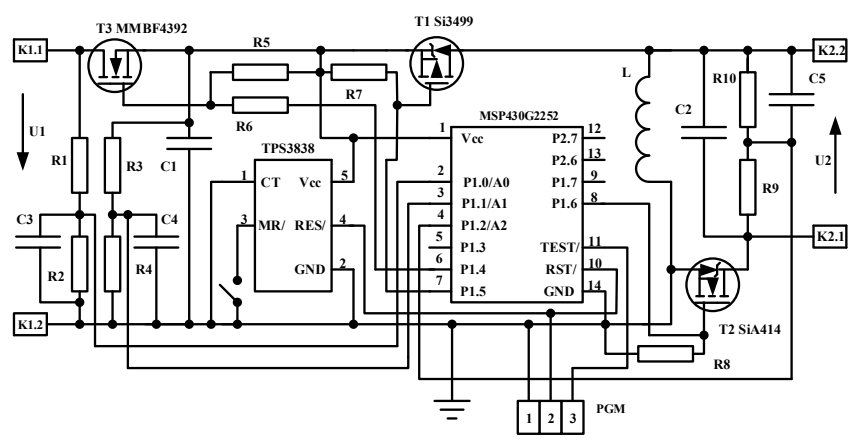

Fig. 5. Energy management system schematic

Output from EH system (photovoltaic cell, thermo generator, vibration generator) is connected to connector $K 1$, which has voltage $U 1$. The supplied device - a sensor node - is connected to connector $K 2$. The energy is stored in supercapacitor $C 1$, alternatively in rechargeable battery. Control unit, which is based on MCU MSP430G2252 enables by energy flow between primary supercapacitor $C 1$ and secondary supercapacitor $C 2$ in both directions by properly switched transistors $T 1$ and $T 2$. The control unit (the MCU) is able to measure:

- Voltage on generator output with no load or with load according to state $T 3$ (input $A 0$ ),

- Voltage $U 1$ on supercapacitor $C 1$ (input $A 1$ ),

- Voltage $U 2$ on supercapacitor $C 2$ (input $A 2$ ).

Based on measured voltages and character of EH energy processor, the energy can be moved between energy storages $C 1$ and $C 2$ to obtain maximal efficiency of the whole system and to operate at maximal point of efficiency. It is clear that in such simple electronic circuit solution (Fig. 5) the system is able to work correctly only if voltage on supercapacitor $C 1$ is in range $2.5 \mathrm{~V}-3.6 \mathrm{~V}$ (supply voltage of the $\mathrm{MCU}$ ) that imposes relatively serious restriction. Though, this problem is able to be solved quite easily - control MCU power supply is separated, alternatively has its own supercapacitor with charge pump from $C 2$.

The Fig. 6 shows voltage behavior at the output of a capacitor $C 1(1000 u F / 16 V)$. The start of the energy pumping is colored white, the voltage at the gate of the upper transistor is colored in cyan and the voltage at lower transistor is colored in purple. The upper transistor is a P-channel so that it is closed 
at high voltage and open at low. Lower transistor is N-channel so it opens at high voltage and closes at low. Input source is a constant voltage of $3.6 \mathrm{~V}$, which is able to deliver up to $500 \mathrm{~mA}$ average current. The process of energy flow starts with opening of the upper transistor for 800us. Immediately, lower transistor opens the same time. During testing, the interval between such "open-closes" was $9.6 \mathrm{~ms}$ and the whole period took $10.4 \mathrm{~ms}$

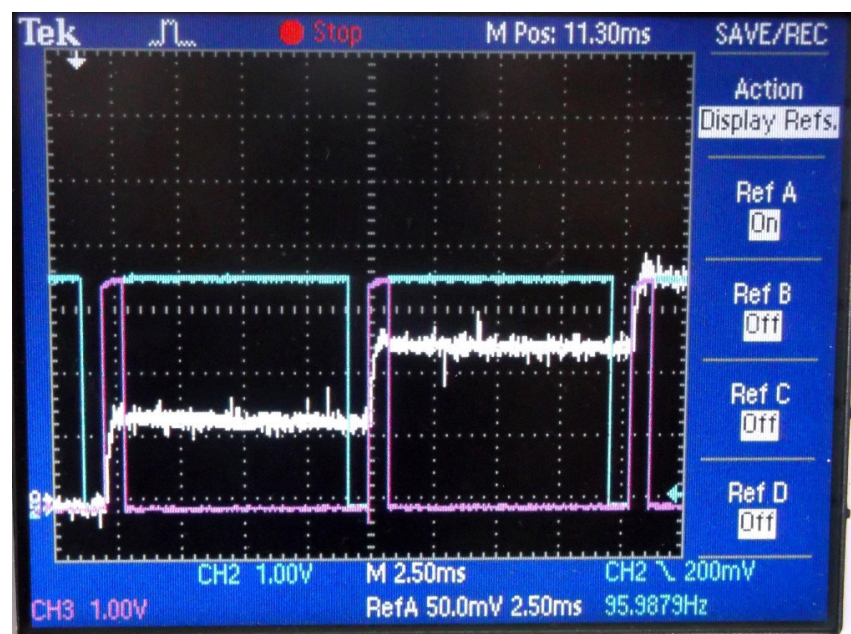

Fig. 6. Voltage behavior of the capacitor $C 1$ on the testbed

The Fig. 7 shows a scenario with $C 2$ capacitor and its alternating charges and discharges. Charging starts with opening of the upper output transistor for 800us following with opening of the lower transistor for $8 \mathrm{us}$. After a short pause $4.1 \mathrm{~ms}$ inverse energy flow takes place $(C 2$ to $C 1)$.

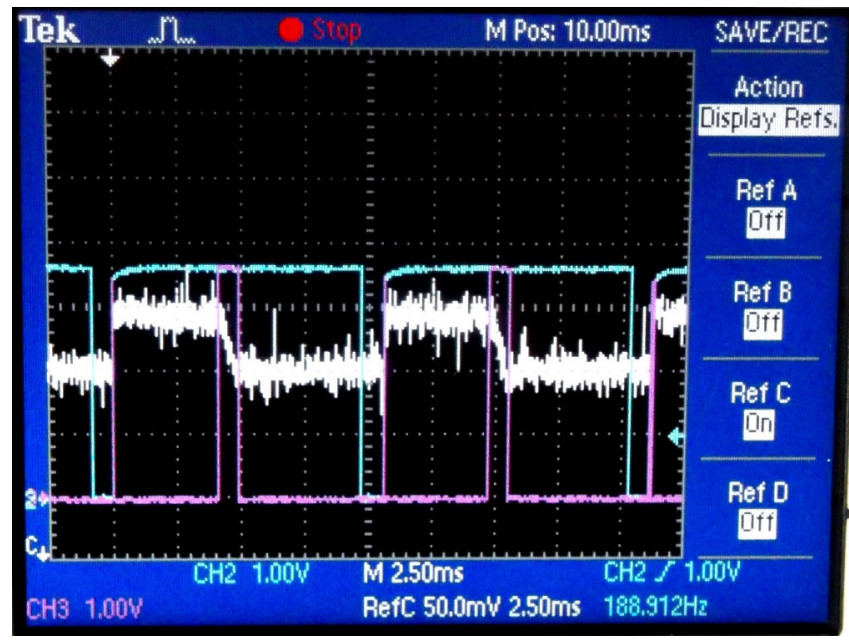

Fig. 7. Voltage behavior at $C 2$ with alternating charges and discharges

The whole cycle takes $10.412 \mathrm{~ms}$. The figure shows steady state of the oscillation and its lower voltage level is $360 \mathrm{mV}$ and its top level reaches $406 \mathrm{mV}$.

It is possible to achieve interesting solutions by connecting this systems from Fig. 2 in series. Alternatively, modifying the mentioned scheme for various EH systems that load power to a joint primary supercapacitor $C 1$. In the latter case the voltage value has to be kept in order to ensure each system to operate in the point of maximal power. As an example, we can speak about more solar panels with various characteristics, alternatively with different exposure, whose energy is stored in a single accumulator cell.

\section{CASE StUdy}

In this section we propose one simple example of the proposed system utilization. Let's imagine the proposed device to be used for energy harvesting purposes from a photovoltaic (PV) module. PV generators have two basic drawbacks i) conversion efficiency of the incident radiation to electric energy is low $(10-20 \%)$, especially at low light intensities; ii) and the amount of the obtained energy depends on the weather. Other disadvantage of solar cells is nonlinear VI characteristic that is changing with the radiation intensity and the temperature. At particular, having the value of light exposure and the temperature on V-I characteristic, it is possible to find just one point, in which PV system works with maximal efficiency. This point is called Maximum Power Point (MPP). For the illustration, the V-I characteristic of a solar module is depicted in Fig. 8.

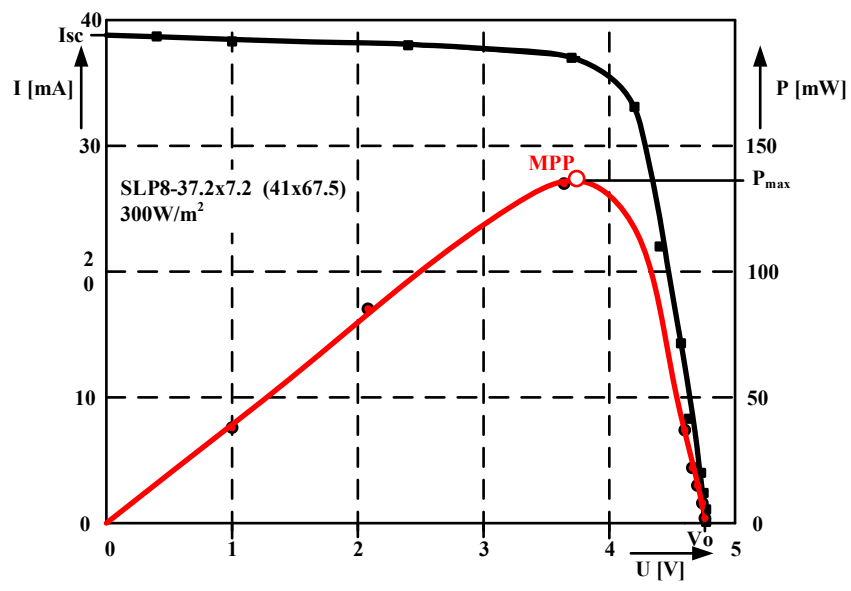

Fig. 8. V-I characterisitcs of solar module

It is clear that during active photovoltaic EH system operation there are various outer conditions. The intensity and angle of incident radiation changes as well as the temperature of solar cells, etc. These conditions considerably influence the parameters of the solar panel as well as the electric energy generator. The aim of the user is a system capable of utilize/accumulate solar energy at the MPP, while changing conditions apply. For this purpose, there are techniques called Maximum Power Point Tracking (MPPT) ensuring that in changes of working conditions, the photovoltaic system (PVS) is able to monitor Maximum Power Point (MPP) and then to ensure the effective operation of the whole system. Problem of designing and evaluation of MPPT methods was dedicated in many papers in specialized journals [22], [23], [24]. At present, there 
is a large number of used methods. In Table ?? there are most often used methods listed and at the same time there is listed its effectiveness as percentage of theoretical reachable value to obtained energy. It is obvious, that listed values are dependent on character of changes in outer conditions, so the table gives average values obtained in 14 different modes. Further information can be found in [22]. Note, that several methods was in [22] tested with various modifications, so Table ?? summarizes the best achieved results in that cases.

TABLE III

MPPT MEHODS AND THEIR EFFECTIVENESS

\begin{tabular}{|l|c|}
\hline \multicolumn{1}{|c|}{ MPPT technique } & Effectiveness [\%] \\
\hline Constant Voltage Method (CV) & 79.5 \\
\hline Short Current Pulse Method (SC) & 90.7 \\
\hline Open Voltage Method (OV) & 94.6 \\
\hline Perturb and Observe Method (P\&O) & 99.3 \\
\hline Incremental Conductance Method (IC) & 99.5 \\
\hline Temperature Method (TP) & 97.1 \\
\hline
\end{tabular}

For the implementation purposes into the proposed system, OV method is used. This method is easy to implement without other demands on the technical equipment. Average effectiveness of solar system with use of this method is sufficient enough. Note, that it is possible to use also other of the above methods, but implementation of MPPT techniques is not the matter of this contribution.

Algorithm of PV system control is the following:

1) Switch off the $T 3$.

2) Delay $10 \mu \mathrm{s}$

3) Voltage with no load measurement $U 1$.

4) Switch on $T 3$.

5) Voltage calculation $U_{p}=0.76 * U 1$.

6) Voltage measurement $U_{C 1}$.

7) Comparison with $U_{p}$, if $\mathrm{U}_{\mathrm{C} 1}>\mathrm{U}_{\mathrm{p}}+\varepsilon$ is true, then pumpU $_{\mathrm{C} 1}()$ if $U_{C 1}<U_{p}$ is true, then $\operatorname{pumpU}_{\mathrm{C} 2}()$

8) Delay $100 \mathrm{~ms}$

9) Go to 1

pumpU $\mathrm{U}_{\mathrm{C} 1}()$ is a function that pumps the energy from supercapacitor $C 1$ to $C 2$ unless the condition is true $\mathrm{U}_{\mathrm{C} 1}>\mathrm{U}_{\mathrm{p}}+\varepsilon$ (opens and closes $T 1$ for the time according to (1) and opens and closes $T 2$ for the time in accordance with (2)), then measures $U_{C 1}$ and compares it with $\mathrm{U}_{\mathrm{p}}+\varepsilon$ and if the condition is met then ends, otherwise repeats the whole "main loop".

pumpU $\mathrm{U}_{\mathrm{C} 2}()$ is a function that pumps the energy from supercapacitor $C 2$ to $C 1$ unless the condition is true $\mathrm{U}_{\mathrm{C} 1}>\mathrm{U}_{\mathrm{p}}$ (opens and closes $T 2$ for the time in accordance with (5) and opens and closes $T 1$ for the time in accordance with (6), then measures $U_{C 1}$ and compares it with $U_{p}$ and if the condition is met then ends, otherwise repeats the whole "main loop".

$\varepsilon$ is the constant, which value depends on values of supercapacitor $C 1$, it constrains oscillation and often pumping of the energy.

In described algorithm, as the source of the energy was used the solar module SLP8-37,2x7,2 with dimensions $41 \times 67,5$ mm. Solar module consists of 8 solar cells that are connected in series. In good working conditions it can generate voltage $U 1$ (with no load) that exceeds allowed operating voltage of the MCU. For this reason it is necessary to ensure that $\mathrm{U}_{\mathrm{C} 1}<3.6 \mathrm{~V}$.

The described example demonstrates applicability of the proposed control unit in EH WSN applications with utilization of solar energy. It is obvious that effectiveness of the solution, as the proportion of obtained energy to theoretical maximum is dependent on specific conditions. Because self-energy consumption of the control unit is constant, it does not depend upon amount of generated energy by the solar module. But this effectiveness also depends upon the size of the solar module and the light exposure.

\section{CONCLUSION}

The control unit of EH system that was developed at our department is designed for controlling the power unit with low power. That fact predetermines it for use in WSN applications, where we expect, that individual nodes are supplied through EH systems and contain energy storages based on supercapacitors, alternatively rechargeable batteries. Control unit, thanks to its versatility and simple parametrization with use of programmable resources, can find its enforcement in all cases, where the realization of effective energetic management with low cost is needed. Despite the fact, that experiments were made only with solar module, it is possible to use the proposed system also in many another applications, where it is needed to implement the bi-directional flow of electric energy.

\section{ACKNOWLEDGMENT}

This contribution/publication is the result of the project implementation Centre of excellence for systems and services of intelligent transport I. ITMS 26220120028 and Research Centre of University of Žilina ITMS 26220220183 supported by the Research \& Development Operational Programme funded by the ERDF.

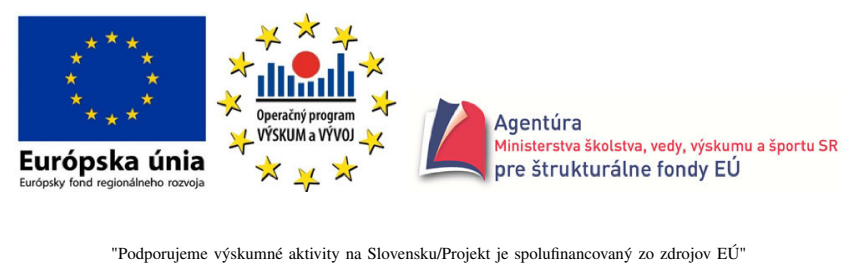

\section{REFERENCES}

[1] S. Abdelwahab, et. al. "Enabling smart cloud services through remote sensing: An internet of everything enabler", Internet of Things Journal, IEEE 1.3 (2014): 276-288. DOI: 10.1109/JIOT.2014.2325071

[2] J. Bradley, J. Barbier and D. Handler, "Embracing the Internet of Everything to Capture Your Share of \$14.4 Trillion,” White Paper, Cisco, 2013.

[3] J. Greenough, "The Internet of Everything," www.businessinsider/internet-of-everising-2015-bi-2014-12?op=1

[4] M. Chovanec, M. Hodon and L. Cechovic, "Tiny low-power WSN node for the vehicle detection", Informatica : an international journal of omputing and informatics. ISSN 0350-5596. Vol. 38, no. 3 (2014), pp. 223-227. 
[5] J. Micek and M. Kochlan, "Energy-efficient communication systems of wireless sensor networks", Studia informatica universalis. ISSN 16217545. Vol. 11, no. 1 (2013), pp. 69-86.

[6] J. M.Gilbert and F. Baluochi, "Comparison of Energy Harvesting Systems for Wireless Sensor Networks", International Journal of Automation and Computing, October, 2008. DOI: 10.1007/s11633-008-0334-2

[7] B. Al-Ghamdi, M. Ayaida and H. Fouchal, "A dynamic slot scheduling for wireless sensors networks", 2014 IEEE Global Communications Conference, GLOBECOM 2014, art. no. 7036834, pp. 357-361. DOI: 10.1109/GLOCOM.2014.7036834

[8] W. K. G. Seah, et. al., "Wireless sensor networks powered by ambient energy harvesting (WSN-HEAP) - Survey and challenges", Wireless Communication, Vehicular Technology, Information Theory and Aerospace \& Electronic Systems Technology, 2009. Wireless VITAE 2009. 1st International Conference, pp.1-5, 17-20 May 2009. DOI 10.1109/WIRELESSVITAE.2009.5172411

[9] S. Grady, "Powering Wearable Technology and Internet of Everything Devices", Cymbet Corporation, www.cymbed.com, 2014.

[10] H. Fouchal, O. Zytoune and D. Aboutajdne, "A battery recovery aware routing protocol for Wireless Sensor Networks", International Symposium on Computers and Communications, Workshops, art. no. 6912636 , DOI: 10.1109/ISCC.2014.6912636

[11] P. Sevcik and O. Kovar, "Power unit based on supercapacitors and solar cell module", SCIECONF 2013 : the 1st international virtual scientific conference, 10.-14. June. ISSN 1339-3561. 2013. ISBN 978-80-5540726-5. pp. 468-471.

[12] T. Bernard, et. al., "Impact of routing protocols on packet retransmission over wireless networks", IEEE International Conference on Communications, art. no. 6654996, pp. 2979-2983. DOI: 10.1109/ICC.2013.6654996

[13] O. Karpis, "Wireless sensor networks in intelligent transportation systems", International journal of modern engineering research (IJMER 2012), ISSN 2249-6645. Vol. 3, iss. 2 (2013).
[14] J. Micek and O. Karpis, "Wireless sensor networks - design of smart sensor node", ICMT'11 : proceedings of the international conference on military technologies 2011. Brno, Czech Republic, 10.-11. May 2011. ISBN 978-80-7231-787-5. pp. 1109-1116.

[15] P. Dutta, et al., "A Building Block Approach to Sensornet Systems," In Proc. SenSys 08, North Carolina, USA, 2008.

[16] Z. Feng, et al., "Collaborative signal and information processing: an information-directed approach", Proceedings of the IEEE, vol.91, no.8, pp.1199-1209, Aug. 2003. DOI: 10.1109/JPROC.2003.814921

[17] O. Karpis, "FFT on ARM-based low-power microcontrollers", International journal of engineering research and development (IJERD). - ISSN 2278-800X. - Vol. 6, no. 9 (2013), pp. 22-26.

[18] M. Kochlan and P. Sevcik, "Supercapacitor power unit for an eventdriven wireless sensor node", Computer Science and Information Systems (FedCSIS), 2012 Federated Conference, pp.791-796, 9.-12. Sept. 2012

[19] S. G. Burrow and P. D. Mitcheson, "Power Conditioning for Energy HarvestingâĂŞTheory and Architecture", Micro Energy Harvesting 5 (2015).

[20] EnerChip EP Energy Processor, www.cymbed.com/pdfs/DS-7215.pdf

[21] P. Sevcik and O. Kovar, "Alternative energy sources for WSN node power supply", ITS 2013 - Intelligent transportation systems 2013. August 26-30, 2013. ISSN 1339-4118. ISBN 978-80-554-0763-0. pp $146-149$.

[22] R. Faranda and S. Leva, "Energy comparison of MPPT techniques for PV Systems," WSEAS Transactions on Power Systems, 6. 2008, ISSN 1790-5060.

[23] D.Freeman, "Introduction to Photovoltaic Systems Maximum Power Point Tracking," Application Report, Texas Instrument, November 2010.

[24] N.Femia, G. Petrone, G. Spagnuolo and M. Vitelli, "Optimization of Pertrub and Observe Maximum Power Point Tracking Method," IEEE Transactions on Power Electronics, vol.20 No.4, July 2005. 